\title{
El Surco Palato Radicular: \\ Su relación con patología pulpar y/o periodontal
}

\author{
PULGAR ENCINAS, R.M.* \\ NOGUEROL RODRIGUEZ, B.**
}

Pulgar Encinas, R.M.; Noguerol Rodriguez, B.El surco palato-radicular: su relación con patología pulpar y/o periodontal. Av Odontoestomatol. 2000; 12, 2: 83-89

\begin{abstract}
RESUMEN
La presencia de anomalías morfológicas radiculares pueden actuar como factor predisponente para el acúmulo de placa y el desarrollo de patología periodontal y/o pulpar. Presentamos un caso clínico de surco palatoradicular en el 21, donde la presencia del surco ha sido el factor causal de una bolsa periodontal con defecto óseo aislado que finalmente condujo a la pérdida del diente. Se analiza la prevalencia, localizaciones, patogenia y la actitud terapéutica correcta ante la presencia de este tipo de anomalías, en base a los datos recogidos de la literatura.
\end{abstract}

\section{PALABRAS CLAVE:}

Surco palato-radicular; sindesmo corono-radicular; anormalidades radiculares; periodontitis localizada.

\section{INTRODUCCIÓN}

A veces el odontólogo y estomatólogo general se encuentra con problemas que suponen un reto a su capacidad diagnóstica. Las anomalías y malformaciones dentarias constituyen uno de los campos de la Odontología donde suele suceder; por una parte, por la prevalencia de este tipo de lesiones (a veces muy baja) y, por otra, por el hecho de que a veces no dan lugar a sintomatología, siendo su diagnóstico anecdótico en el historial clínico del paciente. Sin embargo, en otras ocasiones se manifiestan de forma desconcertante impidiendo el diagnóstico temprano y correcto del problema. La clínica, en estos casos, puede ser pulpar $\circ$ incluso periodontal, siendo refractaria al tratamiento habitual al no haber sido detectada la causa primaria del problema. El clínico observa con perplejidad que tratamientos técnicamente correctos no conducen al éxito esperado sin que aparentemente exista causa que lo justifique. La superposición de problemas puede conducir a retratamientos sin ninguna expectativa de éxito. Además, se da la circunstancia que la publicación de estos casos clínicos donde se aborda el problema diagnóstico y terapéutico de estas anomalías se realiza en revistas de especialidad, habitualmente no seguidas por el clínico general, lo que motiva que éste no las considere en sus esquemas diagnósticos habituales. El diagnóstico de estas lesiones resulta de interés en la medida que condiciona tanto la pauta terapéutica como su propio pronóstico, y conlleva implicaciones periodontales, endodónticas e incluso restauradoras (p.e. prótesis fija) dada su influencia sobre la viabilidad a largo plazo del propio diente.

Tal es el caso de la anomalía conocida en la literatura como surco palato-radicular. Descrita por primera vez como fuente potencial de problemas clínicos en 1965 (1), aunque Mitchell ya lo había descrito en 1892 como "proceso con forma de asta", ha recibido numerosas denominaciones, tales como surco palatogingival, surco distolingual (2), distopalatino (3) o radicular lingual (4), anomalía radicular del desarrollo o anomalía de Chompret (5). Si bien hay escasa bibliografía al respecto comunicando casos clínicos, su referencia suele ser habitual en los tratados de Periodoncia (2,3,6) y Endodoncia (7), lo que resulta orientativo en relación con las implicaciones clínicas y terapéuticas de esta patología.

* Prof. Asociado de Patología y Terapéutica Dental. Departamento de Estomatología. Facultad de Odontología Universidad de Granada.

** Prof. Asociado de Odontología Integrada de Adultos. Departamento de Estomatología. Facultad de Odontología Universidad de Granada. 


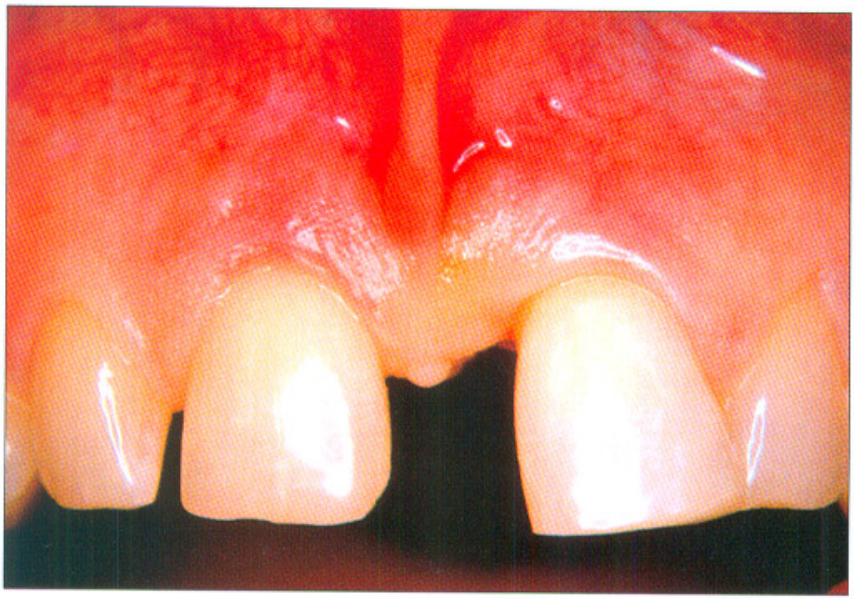

Fig. la.: Vista vestibular del 21. gran desplazamiento disto-vestibular.

El surco palato-radicular es una anomalía en el desarrollo que suele afectar a incisivos superiores aunque también se ha descrito en inferiores (6). Este defecto consiste en una invaginación que aparece en la superficie lingual radicular, en la zona mesial, mediopalatina o distal, en la unión del cíngulo con los rebordes marginales, extendiéndose en grado variable en dirección apical. De forma excepcional, el surco puede aparecer en la cara vestibular $(8,9)$.

En este trabajo presentamos un caso clínico que ilustra esta patología, e intentaremos analizar las implicaciones diagnósticas y terapéuticas que conlleva.

\section{CASO CLÍNICO}

\section{Diagnóstico}

Paciente varón de 34 años que acude a la consulta por presentar su incisivo central izquierdo (21) un desplazamiento vestíbulo-distal muy marcado (fig. 1 a y b). $\mathrm{El}$ paciente no refiere sintomatología de ningún tipo, habiendo acudido a la consulta por las implicaciones estéticas que el desplazamiento produce. A la exploración se comprueba la ausencia de caries, trauma oclusal y las pruebas de vitalidad pulpar son normales. Se comprueba efectivamente la movilidad del diente y su desplazamiento y ante la presencia de una bolsa periodontal mesial profunda se decide remitirlo al periodoncista.

La exploración periodontal del paciente mostraba la presencia de bolsas de 5-6 mm en las regiones molares y menores en el resto de la boca. En mesio-vestibular del 21 la profundidad de sondaje era de $9 \mathrm{~mm}$ y en mesio-palatino de $10 \mathrm{~mm}$ (Fig. 2). El resto de los

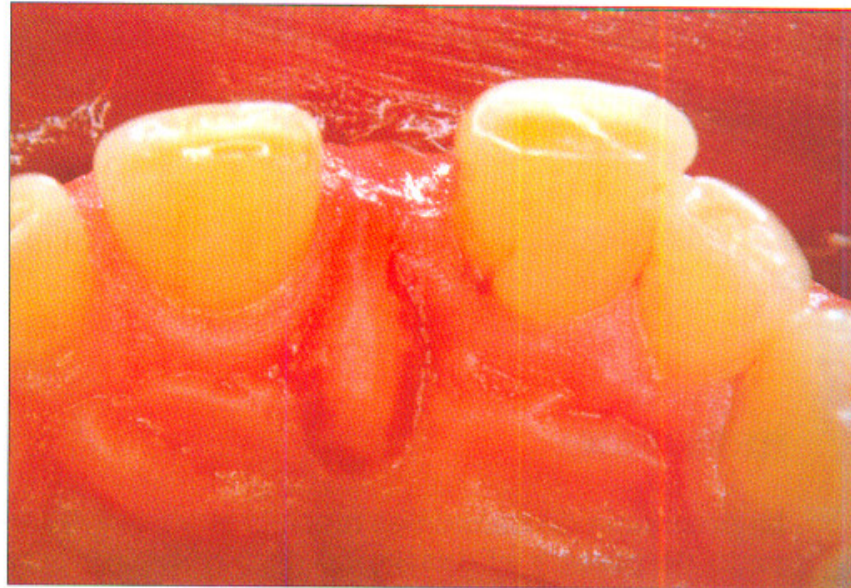

Fig. 1b: Visión palatina del 21. se observa la presencia del surco palato-radicular.

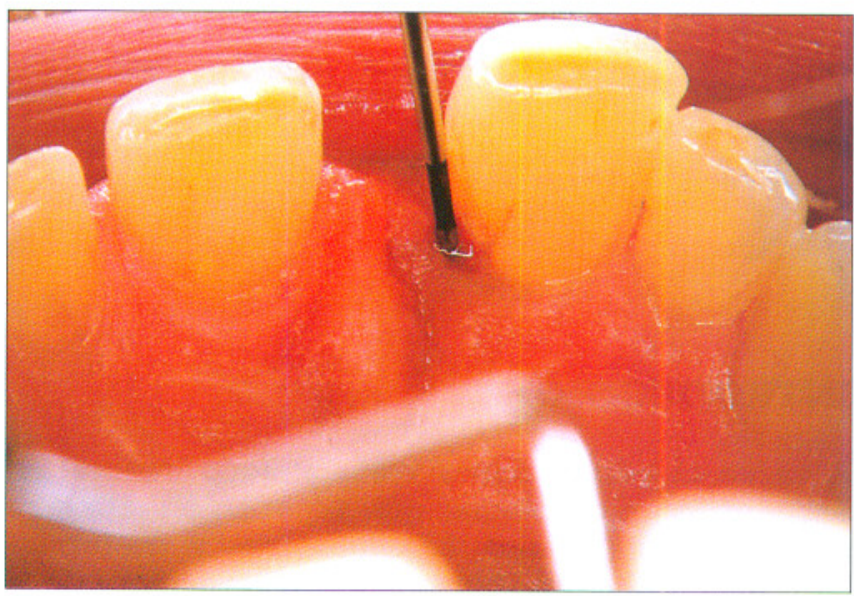

Fig. 2: Sondaje. Se comprueba la existencia de bolsa periodontal en mesial del 21.

sondajes del diente mostraba una profundidad inferior a $10 \mathrm{~mm}$.

El contacto en céntrica era inexistente así como en los movimientos de lateralidad y protusiva. Se verificó la ausencia de frémito. En deglución se observaba el empuje lingual en incisivos y la interposición en la zona del diastema.

La exploración radiológica mediante serie radiográfica completa mostró una pérdida incipiente con áreas de pérdida moderada en zonas molares y un gran defecto en mesial del 21 con nivel de hueso normal en distal. El 11 no presentaba ningún nivel de pérdida ósea. (Fig. 3).

Los niveles de higiene oral eran aceptables con un $21 \%$ de superficies con placa y un IP\% de 1,12. El porcentaje de superficies con sangrado al sondaje fue del $8 \%$ con sangrado abundante en mesial del 21 


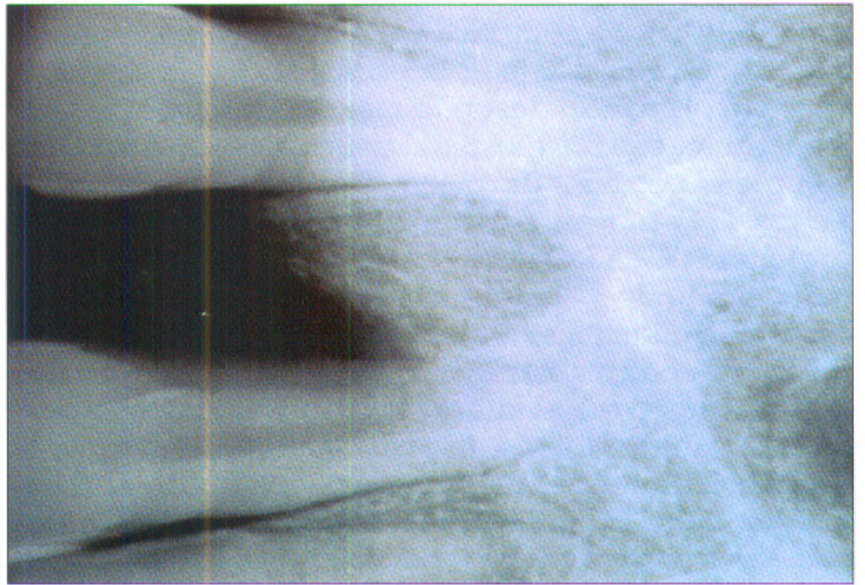

Fig. 3: Rx periapical de 21 que muestra el gran defecto óseo mesial.

y ausencia de hemorragia en 11 y 22 . El IG\% fue de 0,71 .

\section{Tratamiento}

Tras la realización de una fase periodontal básica mediante raspado y alisado radicular se reevaluó el caso persistiendo una profundidad de sondaje en la zona problema del 21 de $6 \mathrm{~mm}$ en MV y de $7 \mathrm{~mm}$ en MP, con sangrado abundante al sondaje.

Se realizó un colgajo mediante incisiones intrasulculares y preservación de papila, pensando en la posibilidad de realizar cirugía regenerativa en la zona; pero la exploración intraoperatoria mostró la presencia de un surco de extensión hasta el ápice con un defecto óseo en dos paredes (Fig. 4).

Se intentó una radiculoplastia mediante cincel, con

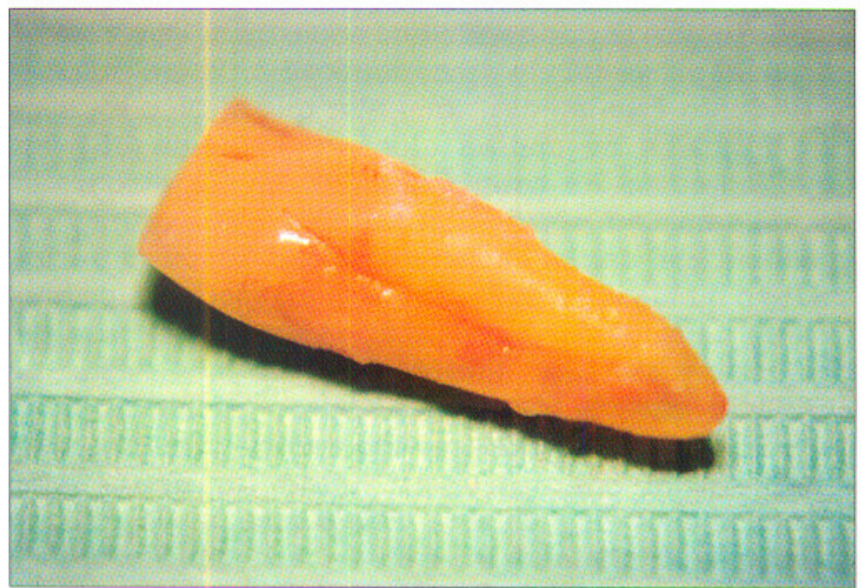

Fig. 5: Extracción del 21. Se comprueba la extensión del surco hasta el ápice.

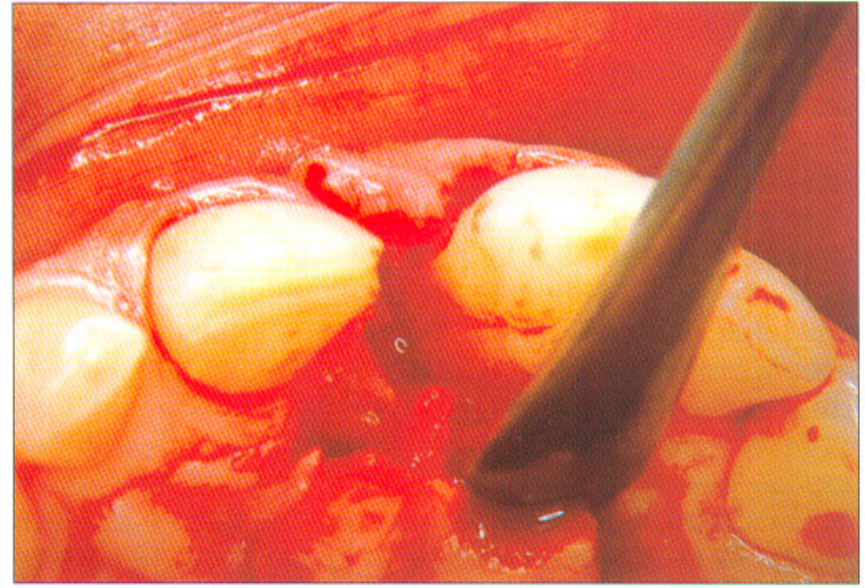

Fig. 4: Fase quirúrgica. Surco palato-radicular que se extiende hasta el ápice con un defecto óseo en dos paredes.

intención de eliminar el surco transformándolo en una depresión abierta. Sin embargo, al realizarla se puso de manifiesto la enorme profundidad del mismo, lo que imposibilita la cirugía regenerativa por la persistencia de la entrada de bacterias a la zona. Finalmente se decidió la extracción del diente, lo que permitió la confirmación del diagnóstico intraoperatorio. (Fig. 5).

Tras un tiempo de espera de 5 meses, se colocó un implante osteointegrado tipo Bränemark, cuyas fases quirúrgica y protética transcurrieron sin incidentes (Fig 6).

\section{DISCUSIÓN}

Los estudios que pretendieron establecer la preva-

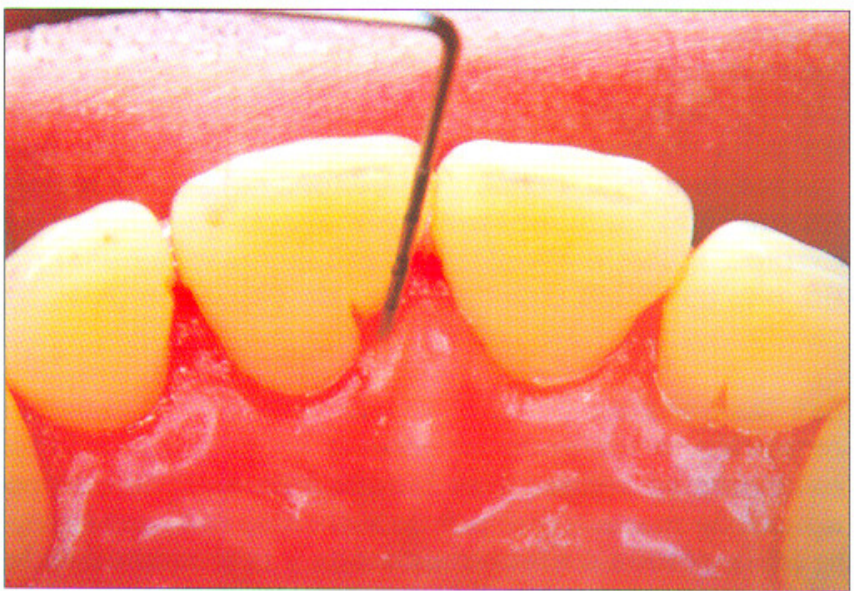

Fig. 6: Surco palato-radicular en mesial de 11. Paciente asintomático. Ausencia de bolsa periodontal. 
lencia de esta anomalía arrojan resultados variables. Everett y Kramer (10) la encontraron en el 1.9\% de los incisivos laterales superiores en una serie de 625 dientes extraídos. Kogon (11) valoró la prevalencia, localización y extensión vertical de surcos palatoradiculares en 3168 incisivos superiores extraídos y encontró que el $5.6 \%$ de los laterales superiores y el $3.4 \%$ de los centrales tenían un defecto; en los laterales, la localización más frecuente era la zona media y el resto se repartían por igual entre mesial y distal, mientras que en el caso de los centrales, los defectos se situaban por igual en las superficies media y mesial, mientras que eran menos frecuentes en la distal. La extensión apical del surco medida desde la unión amelocementaria fue para los laterales de 1 a $5 \mathrm{~mm}$ en el $43 \%$ de los dientes, de 6 a 10 $\mathrm{mm}$ en el $47 \%$ y de más de $10 \mathrm{~mm}$ en el resto. Las cifras correspondientes a los centrales fueron de 1 a $5 \mathrm{~mm}$ en el $39 \%$, de 6 a $10 \mathrm{~mm}$ en el 37 y de más de $10 \mathrm{~mm}$ en el $24 \%$.

Dos estudios han evaluado la presencia de surcos palatoradiculares in vivo. Withers y col (12) determinaron en una serie de 531 militares norteamericanos que el $8,5 \%$ de los pacientes presentaban al menos un incisivo con un surco de desarrollo; con respecto a los dientes lo hallaron en el $4.4 \%$ de los laterales y en el $0.28 \%$ de los centrales. El otro trabajo es el de Hou y Tsai (13), que encontraron que el $44,6 \%$ de los pacientes de su serie ( 101 individuos en total) tenía al menos un incisivo superior con un surco de desarrollo; con respecto a los dientes, el $30,2 \%$ de los laterales y el 5,9\% de los centrales tenían un defecto. Las discrepancias entre las cifras que se barajan en la literatura puede ser debida, por un lado, a diferencias entre los criterios diagnósticos (11); por la diferente distribución entre razas (llaman la atención las cifras de Hou y Tsai), y finalmente, por el tipo de muestras sobre las que se establecen, ya que mientras en algunos trabajos se estudian sobre dientes extraídos $(10,11)$ en otras el cálculo se realiza en grupos de pacientes a los que se realiza un exploración clínica cuidadosa de los incisivos en busca de la anomalía $(12,13)$. En cualquier caso, en ninguno de los escasos trabajos en los que se intenta establecer cuán frecuente es esta alteración las condiciones de la muestra son lo suficientemente rigurosas como para poder extrapolar los resultados a la población general siendo, por tanto, los datos de los que disponemos básicamente orientativos.

Las lesiones se pueden clasificar atendiendo a su extensión o teniendo en cuenta su profundidad. La extensión se refiere a su longitud en sentido corono apical, de manera que pueden situarse exclusivamente en la corona o efectar a corona y raíz ascendiendo en mayor o menor medida por la superficie radicular. Cuanto mayor sea la extensión de la lesión mayor probabilidad de complicaciones endo y periodontales (14). En relación con la profundidad, es bastante variable oscilando desde lo que Gao y cols. (15) califican de un surco superficial del desarrollo hasta una completa pérdida de cierre del tejido calcificado. Kogon (11) por su parte, distingue atendiendo a la profundidad de la lesión, tres grados: superficial $(<1$ $\mathrm{mm})$, profundo (>l mm) o canal en forma de tunel, cuando la comunicación es completa. A veces resulta difícil en clínica diferenciar, sobre todo, los grados más severos, pero el interés de esta clasificación reside básicamente desde un punto de vista clínico, en el establecimiento del pronóstico de respuesta al tratamiento, dada las diferencias existentes entre las formas leves (superficiales y poco extensas) y las graves (más extensas y/o profundas).

En cuanto a la patogenia, la presencia de un surco palatogingival a lo largo de la raiz probablemente es consecuencia de la invaginación del órgano del esmalte y de la vaina epitelial de Hertwig tal como ocurre en el dens invaginatus (dens in dente), pero en este caso la invaginación es de menor extensión (16). Con toda seguridad la inserción epitelial es a este nivel defectuosa, por lo que esta zona se constituye en una vía para el acúmulo de microorganimos con la formación de la subsiguiente bolsa periodontal. Por otra parte, la presencia de túbulos dentinarios expuestos como resultado de procesos de reabsorción en las caras y en la base de las lesiones de tipo profundo, ha sido considerada por algunos autores como el mecanismo patogénico que promueve la entrada de bacterias a la pulpa, tal como sucede con el dens in dente y que explicaría el desarrollo de áreas periapicales en aquellos casos en los que no existe antecedente de caries o traumatismo. Sin embargo, y sin descartar lo mencinado anteriormente, Gao y cols (15) comprobaron mediante SEM la presencia de forámenes accesorios no sólo en las paredes del surco palatogingival sino también en las correspondientes al conducto pulpar radicular comunicados entre sí por conductos accesorios que constituirían la principal vía de comunicación entre pulpa y pariodonto. Finalmente, comentar que si bien los surcos linguales no suelen alcanzar el apex (15), en aquellos casos de gran extensión la vía retrógrada podría también justificar la complicación pulpar en ausencia de caries o trauma (complicación perioendo) o incluso lo contrario, los casos raros en los que los síntomás iniciales son de tipo pulpar, y el paso de bacterias y material necrótico se produce desde el conducto radicular hacia el surco palatogingival constituyéndose en la base inflamatoria que progresa en dirección coronal destruyendo las estructuras periodontales (16). En este último caso, radiográficamente se observa una imagen de radiolucidez apical que progresa en dirección coronal (imagen "en lágrima"). 
El diagnóstico clínico de este tipo de anomalías es fundamental por las implicaciones terapéuticas que acarrea. Un diagnóstico correcto y a tiempo puede ahorar molestias y esfuerzos tanto al dentista como al paciente, evitando la necesidad de tratamientos sucesivos que en muchos casos están condenados al fracaso.

El paciente puede presentar síntomas periodontales, pulpares o incluso estar asintomático. En todo caso, el diagnóstico diferencial se establecerá siempre con problemas pulpares de origen carioso o traumático (ya sea oclusal o de otro tipo) y enfermedad periodontal generalizada, dependiendo de las manifestaciones clínicas concretas del paciente.

Si la situación es de sintomatología puramente periodontal, el diagnóstico puede realizarse mediante exploración siguiendo el surco por debajo del margen gingival y comprobando la presencia de la bolsa correspondiente. Esta bolsa periodontal normalmente es tubular y localizada en la zona radicular en donde se comprueba la presencia del surco en oposición a aquellos casos en los que hay enfermedad periodontal verdadera y generalizada en torno a toda la superficie radicular. Las pruebas pulpares demuestran vitalidad y radiográficamente un área de radiolucidez vertical que sigue el trayecto del surco. Finalmente, en estadíos más avanzados, podría aparecer sintomatología endodóntica.

Si el problema se asocia a complicaciones pulpares el paciente puede presentar este tipo de síntomas en cualquiera de sus variantes, sin que a veces se compruebe la presencia de caries ni historia de traumatismos previos. En este caso el clínico no debe anticiparse y debe comprobar el estado periodontal, que puede ser normal o asociado a bolsa periodontal. La presencia de un área radiolúcida en forma de lágrima debe hacer sospechar la existencia de esta anomalía aunque no existan manifestaciones periodontales, tanto en casos de problema pulpar inicial como en aquellos en los que se hubiera producido fracaso endodóntico (18). En este último caso la existencia de una apertura cameral previa o incluso de una obturación podrían dificultar el diagnóstico a la exploración. A veces, cuando el surco es lo suficientemente profundo, se pude visualizar radiográficamente en forma de una línea negra que recorre la raiz, dando una imagen similar a la que produce la fractura vertical radicular con la que habrá que realizar diagnóstico diferencial (17).

Finalmente, en cuanto al tratamiento, será necesario valorar cuidadosamente la situación clínica concreta del paciente. En general, en este tipo de casos resulta imposible predecir la evolución y, según los resultados recogidos en la bibliografía, éstos pueden ser dispares $(19,20,21,22,23)$. Esta evidencia obliga al clí-

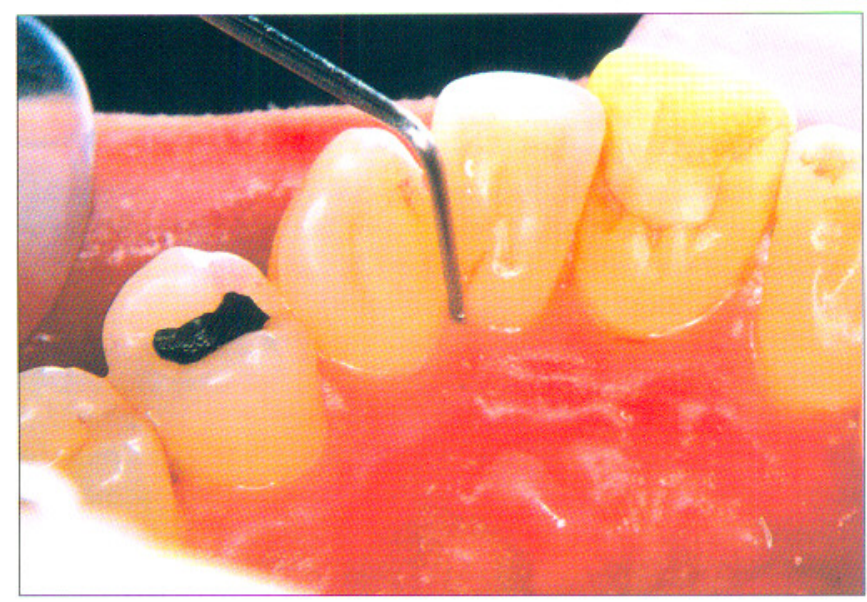

Fig. 7: Surco palato-radicular en distal del 12. Paciente asintomático en protocolo de seguimiento.

nico a considerar cada caso de forma aislada y siempre a recomendar al paciente la pauta que considere más apropiada, pero expresando sus reservas en cuanto al pronóstico.

\section{Paciente asintomático}

Será sometido a un protocolo de seguimiento clínico riguroso. Dado que la existencia de este tipo de defectos se asocia a alteraciones en la salud periodontal de la zona a pesar de que el paciente pueda permanecer asintomático $(12,13)$ (Fig. 7 y 8), será imprescindible una instrucción rigurosa en técnicas de higiene oral para intentar evitar el desarrollo de sintomatología.

\section{Paciente con problemas pulpares exclusivamente}

Se informará al paciente de lo incierto del resultado del tratamiento endodóntico, cuyo éxito dependerá del número y localización de los canales accesorios presentes entre surco y pulpa.

\section{Paciente con manifestaciones periodontales}

Se valorará el estado pulpar. En caso de pulpa viva, se podría adoptar una actitud espectante a la espera de evolución; en el caso de necrosis, se recomienda tratamiento, con información previa al paciente sobre su pronóstico. En cuanto al problema periodontal, se acepta que mientras el anclaje epitelial permanece intacto, el periodonto permenece sano, pero en cuanto ésta fracasa el surco es invadido y puede comenzar la formación de una bolsa infraósea a lo largo de su longitud. Si bien para algunos autores esta situación es clínicamente intratable (17), para otros se valorará la 
posibilidad de tratamiento (odontoplasia+relleno) del defecto teniendo siempre en consideración que el pronóstico se ensombrece conforme aumenta la profundidad y/o extensión del surco. La odontoplastia, alisado radicular agresivo, se realiza para eliminar el cálculo y el cemento afectado y, al mismo tiempo, alisar y aplanar la superficie radicular (24). La persistencia del surco profundo se convierte per se en un factor de recidiva de la lesión independientemente del procedimiento terapéutico aplicado (25). Una vez eliminado el cemento necrótico, nada se opone a la realización de un tratamiento regenerativo mediante técnicas de regeneración tisular guiada, cuando se haya conseguido eliminar la concavidad para garantizar la ausencia de recolonización bacteriana posterior.

\section{SUMMARY}

The presence of radicular anomalies may act as a predisposing factor for the accumulation of bacterial plaque and the development of local periodontal or pulpal disease. We present a case of palato-radicular groove in tooth No. 21 that caused periodontal pocket and localized bone defect leading to loss of the tooth. We review the prevalence, localizations and pathogenesis of this dental abnormality as well as therapeutic approaches reported in the literature.

The palato-radicular groove:relationship with pulpar and/or periodontal desease

\section{KEY WORDS}

Palato-radicular groove; radicular anormalities; localized periodontitis.

\section{CORRESPONDENCIA}

Rosa Pulgar Encinas. Departamento de Estomatología. Facultad de Odontología Universidad de Granada. Colegio Máximo. Campus de Cartuja s/n. 18071. Granada.

\section{BIBLIOGRAFÍA}

1. Prichard, JS. Advanced periodontal theraphy. W.B. Saunders Co.Philadelphia, 1965.
2. Genco, R.J. Periodoncia. Interamericana. MacGraw-Hill. Mejico, 1993.

3. Schluger, S; Youdelis, R; Page, RC; Johnson, RH. Periodontal Diseases. Ed. Lea \& Febiger. Philadelphia. 1990.

4. August, DS. The redicular lingual groove: an overlooked differential diagnosis. JADA 1978 ; 96:1037-39.

5. Nadal-Valdaura, A. Patologia Dentaria. Ed. Rondas. Barcelona 1987.

6. Carranza, FA. Glickman's Clinical Periodontology. W.B. Saunders Company. Philadelphia. 1990.

7. Cohen, S.; Burns, RC. Pathways of the pulp. C.V. Mosby Co. St. Louis. 1976.

8. Kozlovsky, A; Tal, H; Yechezkiely, N; Mozes, O. Facial radicular groove in a maxilary central incisor. J Perodontol 1988; 59: 615-17.

9. Goon WW; Carpenter WM; Brace, NM; Ahlfeld RJ. Complex facial radicular groove in a maxillary lateral incisor. J Endod 1991, 17:244-8.

10. Everett, FG; Kramer, GM. The disto-lingual groove in the maxillary lateral incisor; a periodontal hazard. J Periodontol 1972; 43:352.

11. Kogon, SL. The prevalence, location and conformation of palato-radicular grooves in maxillary incisors. J Periodontol 1986 57: 231-34.

12. Withers, JA; Brunsvold, MA; Killoy, WJ; Rahe, AJ. The relationship of palato-gingival grooves to localized periodontal disease. J Periodontol 1981 52: 41-44.

13. Hou, GL; Tsai, CC. Relationship between palatoradicular grooves and localized periodontitis. J Clin Periodontol 1993 20:678-82.

14. Gher, ME; Vernino, AR. Root morphology- Clinical sigificance in pathogenesis and treatment of periodontal disease. JADA 1980 101:627-33.

15. Gao, Z; Shi, J; Wang, Y; Gu, F. Scanning electron microscopic investigation of maxillary lateral incisors with a radicular lingual groove.Oral Surg Oral Med Oral Pathol 1989; 68:462-6.

16. Lee, KW; Lee, EC; Poon, KY. Palato-gingival grooves in maxillary incisors. Br Dent J 1968 2: 14-18.

17. Simon, JHS; Glick, DH; Frank, AL. Predictable endodontic and periodontic failures as a result of radicular anomalies. Oral Surg 1971;31:823-26. 
18. Fabra Campos, H. Failure of endodontic treatment due to a palatal gingival groove in a maxilary lateral incisor with talon cusp and two root canals. J Endod 1990; 16:342-5.

19. Holen, S. The radicular groove. (Letter). JADA 1978 97:441.

20. Peikoff, MD; Perry, JB; Chapipnick, LA. Endodontic failure attributable to a complex radicular lingual groove. J Endod 1985 11:573-7.

21. Kozlovsky, A; Tal,H; Yechezkiely, N Mozes, O. Facial radicular groove in a maxilary central incisor. A case report. J Periodontol 59:615-7.
22. Hosomi, T; Yoshikawa, M; Yaoi, M; Sakiyama, Y; Toda, T. A maxillary central incisor having two root canals geminated with a supernumerary tooth. J Endod 1989 15:161-3.

23. Jeng, JH; Jackson-Lu HK; Hou, LT. Treatment of an osseous lesion associated with a severe palato-radicular groove: A case report. J Periodontol 1992 63:708-12.

24. Sterrett, JD. Consideraciones estéticas en el tratamiento de defectos periodontales asociados a surcos palatoradiculares bilaterales. J Esthet Dent 6:12-19.

25. Löe $H$. The Gingival Index, the Plaque Index and the Retention Index systems. J Periodontol 1967; 38:610-6. 
